\title{
Overlap hypercube fermions in QCD with light quarks
}

Wolfgang Bietenholz*

Institut für Physik

Humboldt Universität zu Berlin

Newtonstr. 15, D-12489 Berlin, Germany

E-mail: bietenho@physik.hu-berlin.de

Stanislav Shcheredin

Fakultät für Physik

Universität Bielefeld

D-33615 Bielefeld, Germany

E-mail: shcheredephysik.hu-berlin.de

We report on simulation results with overlap hypercube fermions (overlap HF) - a type of exactly chiral lattice fermions - and their link to chiral perturbation theory. We first sketch the construction of the overlap HF and discuss its high level of locality. Next we show applications in the $p$-regime of QCD, where we evaluate $m_{\pi}, m_{\rho}$, the quark mass according to the PCAC relation, the renormalisation constant $Z_{A}$ and the pion decay constant $F_{\pi}$ as functions of the bare quark mass. $F_{\pi}$ is then reconsidered at even smaller quark masses in the $\varepsilon$-regime, along with the scalar condensate $\Sigma$. In that context we also discuss results for the topological charges and susceptibility.

XXIIIrd International Symposium on Lattice Field Theory

25-30 July 2005

Trinity College, Dublin, Ireland

\footnotetext{
*Speaker.
} 


\section{Overlap Hypercube Fermions}

For free fermions, perfect lattice actions are known analytically [1]. The corresponding lattice Dirac operator can be truncated to represent a free Hypercube Fermion (HF), which still has excellent scaling and chirality properties [2]. The HF is gauged by fat links over the shortest lattice paths. Finally the links are amplified by a factor $u \gtrsim 1$ to restore criticality and minimise the violation of the Ginsparg-Wilson relation. Due to the truncation and the imperfect gauging procedure, the scaling behaviour and the chirality are somewhat distorted. Chirality can be corrected again by inserting the $\mathrm{HF}$ in the overlap formula [3] (at lattice spacing $a$ )

$$
D_{\mathrm{ov}}=\frac{\rho}{a}\left(1+A / \sqrt{A^{\dagger} A}\right), \quad A:=D_{0}-\frac{\rho}{a}, \quad \rho \gtrsim 1,
$$

where $D_{0}$ is some lattice Dirac operator with $D_{0}=\gamma_{5} D_{0}^{\dagger} \gamma_{5}$ ( $\gamma_{5}$-Hermiticity).

- The standard overlap fermion is obtained by inserting the Wilson operator, $D_{0}=D_{\mathrm{W}}$, which is then drastically changed. We denote the resulting standard overlap operator as $\mathbf{D}_{\mathbf{o v}-\mathbf{W}}$.

- Here we study the case where the HF is inserted in the overlap formula (11.1), $D_{0}=D_{\mathrm{HF}}$. This yields the operator $\mathbf{D}_{\mathbf{o v}-\mathbf{H F}}$, which describes the overlap $H F$.

In both cases, one arrives at exact solutions to the Ginsparg-Wilson relation, and therefore at an exact (lattice modified) chiral symmetry [ []. ${ }^{1}$ However, in contrast to $D_{\mathrm{W}}, D_{\mathrm{HF}}$ is approximately chiral already, hence its transformation by the overlap formula, $D_{\mathrm{HF}} \rightarrow D_{\mathrm{ov}-\mathrm{HF}}$, is only a modest modification. Therefore, the virtues of the HF are essentially inherited by the overlap HF [7].

Here we are going to show mostly quenched results with the standard gauge action at $\beta=5.85$ (i.e. $a \simeq 0.123 \mathrm{fm}$ ). For details of the overlap HF construction - as well as its locality, which is superior compared to $D_{\mathrm{ov}-\mathrm{W}}$ - we refer to Ref. [8]]

In Fig. 1 we illustrate the locality at strong gauge coupling: at $\beta=5.7$ the standard overlap operator is still barely local, if one chooses the optimal value $\rho=1.8$. Similarly we optimise $u=1.6$ for the HF (at $\rho=1$ ) and we still find a clear locality at $\beta=5.7$, which is in fact stronger than the one observed for $D_{\text {ov-W }}$ at $\beta=6$ (and optimal $\rho$ ) [9].

If we proceed to $\beta=5.6$, the locality collapses for $D_{\mathrm{ov}-\mathrm{W}}$, hence in that case the standard overlap formulation does not provide a valid Dirac operator. On the other hand, if we insert the HF at $u=1.7$ we still observe locality. Thus the overlap-HF formulation provides chiral fermions on coarser lattices.

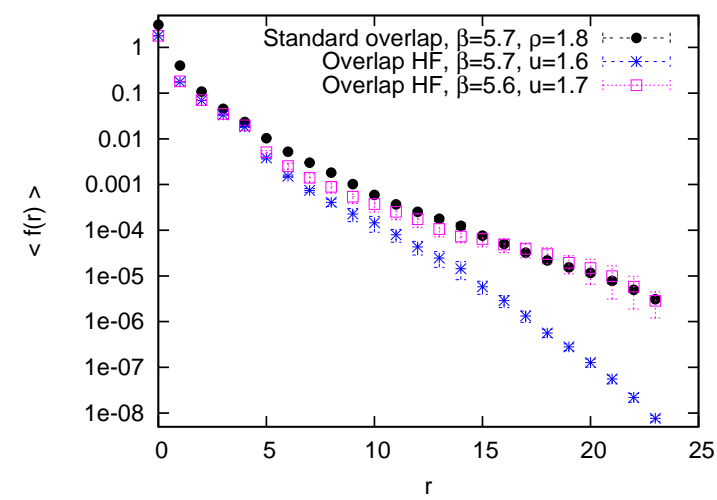

Figure 1: The locality of different overlap fermions, measured by the maximal impact of a unit source $\bar{\psi}_{x}$ on $\psi_{y}$ over a distance $r=\|x-y\|_{1}$. At the same value of $\beta$, the overlap HF is clearly more local, and its locality persists up to $\beta=5.6$, where the standard overlap fermion collapses.

\section{Applications in the $p$-Regime}

We first present results in the $p$-regime, which is characterised by a box length $L \gg 1 / m_{\pi}$, so that the $p$-expansion of chiral perturbation theory $(\chi \mathrm{PT})$ is applicable. We consider $\beta=5.85$, a

\footnotetext{
${ }^{1}$ The correctness of the axial anomaly in all topological sectors has been verified for the standard overlap operator in Ref. [.
} 
lattice of size $12^{3} \times 24$ and (bare) quark masses of $a m_{q}=0.01,0.02,0.04,0.06,0.08$ and 0.1 . We computed 100 overlap HF propagators and first evaluated $m_{\pi}$ in three different ways: (1) From the pseudoscalar correlator $\langle P P\rangle$, where $P=\bar{\psi} \gamma_{5} \psi$ (2) From the axial correlator $\left\langle A_{4} A_{4}\right\rangle$, where $A_{4}=\bar{\psi} \gamma_{4} \gamma_{5} \psi$ (3) From $\langle P P-S S\rangle$, where $S=\bar{\psi} \psi$. The subtraction of the scalar density is useful at small $m_{q}$ to avoid the contamination by zero modes.
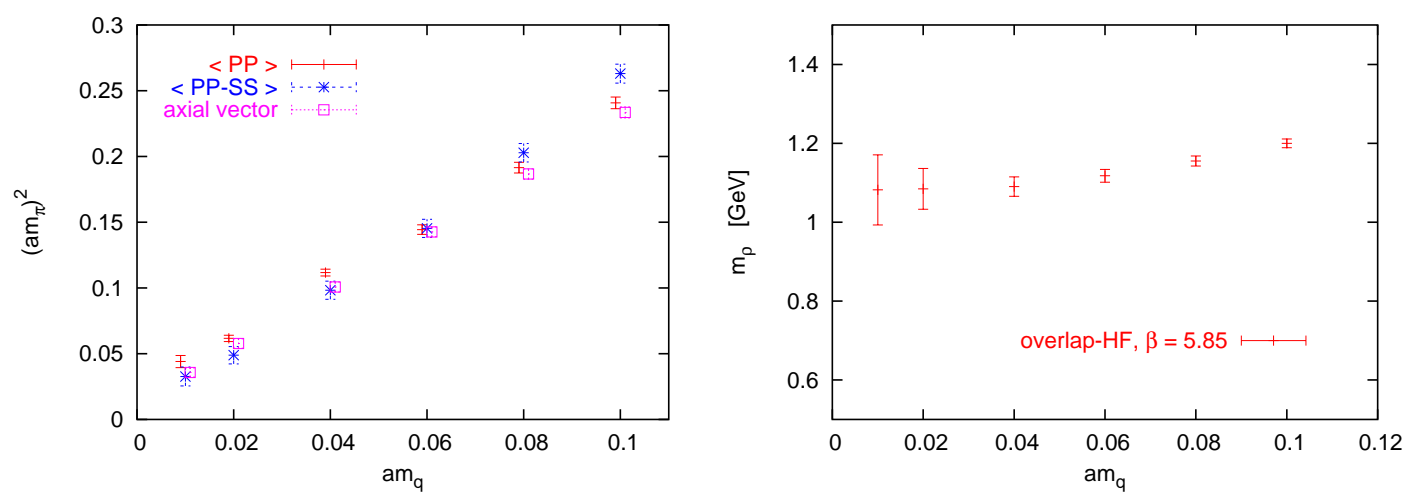

Figure 2: On the left: The pion mass evaluated in various ways. On the right: the $\rho$-meson mass.

The results are shown in Fig. 2 (left): they follow well the expected behaviour $a m_{\pi}^{2} \propto m_{q}$, in particular for $\langle P P-S S\rangle$, with a linearly extrapolated intercept of $a^{2} m_{\pi, P P-S S}^{2}\left(m_{q}=0\right)=0.0001(15)$. The hierarchy at small $m_{q}, m_{\pi, P P}>m_{\pi, A A}>m_{\pi, P P-S S}$, agrees with the literature [10]. Our smallest pion mass has Compton wave length $\approx L / 2$, so we are at the edge of the $p$-regime.

Fig. 目 (right) shows our results for the vector meson mass, with a chiral extrapolation to $m_{\rho}=1017(39) \mathrm{MeV}$ (quenched results tend to be above the physical $\rho$ mass).
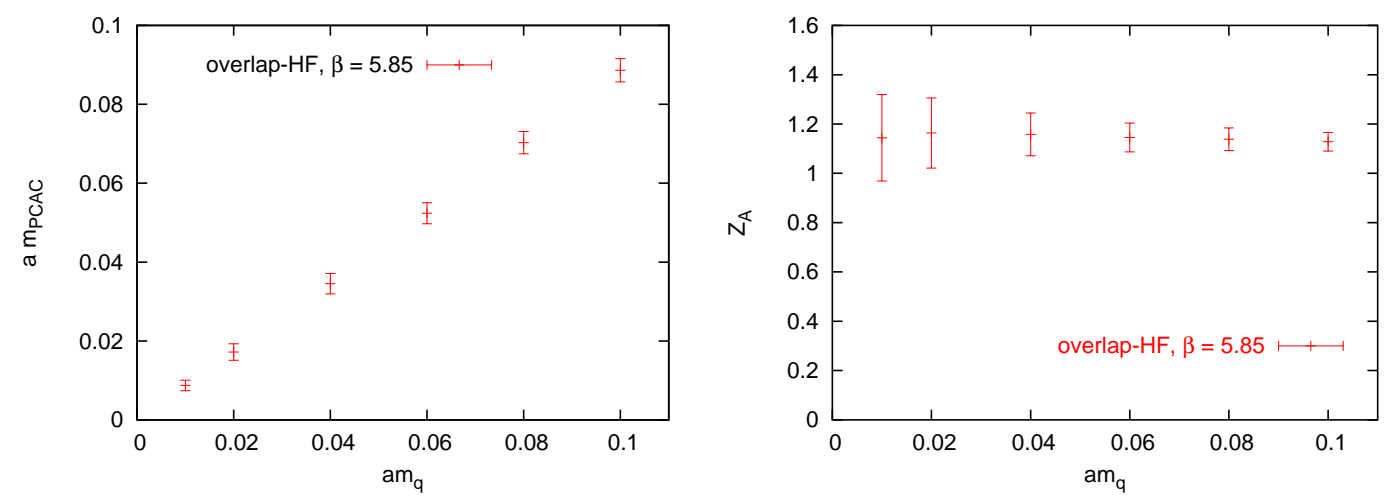

Figure 3: The PCAC quark mass and the renormalisation constant $Z_{A}=m_{q} / m_{\mathrm{PCAC}}$.

In Fig. 3 (left) we consider the quark mass obtained from the axial Ward identity,

$$
m_{\mathrm{PCAC}}=\left[\sum_{\vec{x}}\left\langle\partial_{4} A^{\dagger}(x) P(0)\right\rangle\right] / 2\left[\sum_{\vec{x}}\left\langle P^{\dagger}(x) P(0)\right\rangle\right] .
$$

We observe also here a nearly linear behaviour, with a chiral extrapolation to $\operatorname{am}_{\mathrm{PCAC}}\left(m_{q}=0\right)=$ $-0.00029(64)$. Remarkably, $m_{\mathrm{PCAC}}$ is close to $m_{q}$, which is not the case for $D_{\mathrm{ov}-\mathrm{W}}$ [11]. Consequently the renormalisation constant $Z_{A}=m_{q} / m_{\mathrm{PCAC}}$ is close to 1 ; it has the chiral extrapolation $\mathbf{Z}_{\mathbf{A}}=\mathbf{1 . 1 7}(\mathbf{2})$. This is in striking contrast to the large $Z_{A}$ factors obtained for the standard overlap fermions [13, 11, 12]. According to Ref. [14] the fat link may be helpful for this favourable feature. 
At last we consider $F_{\pi}=\frac{2 m_{q}}{m_{\pi}^{2}}|\langle 0|P| \pi\rangle|$, by using either $P P$ or $P P-S S$, see Fig. $甘$. The extrapolation to $m_{q}=0$ yields $F_{\pi, P P}=111.5(2.5)$ $\mathrm{MeV}$, resp. $\quad \mathbf{F}_{\pi, \mathbf{P P}-\mathbf{S S}}=\mathbf{1 0 4}(\mathbf{9}) \mathbf{M e V}$, which is above the physical value (taken to the chiral limit) of $86 \mathrm{MeV}$ [15]. In particular the behaviour of the $\langle P P-S S\rangle$ result at small $m_{q}$ motivates us to reconsider $F_{\pi}$ at yet smaller quark masses, which takes us to the $\varepsilon$-regime.

\section{Applications in the $\varepsilon$-Regime}

In the $\varepsilon$-regime [16] the correlation length

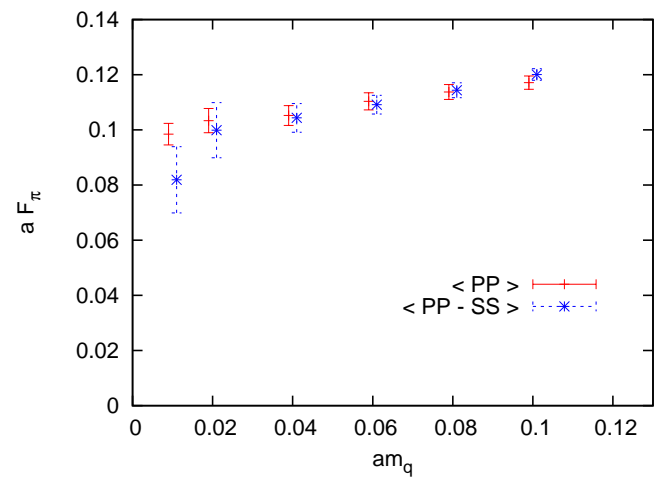

Figure 4: The pion decay constant from a direct evaluation in the $p$-regime, using the overlap HF.

exceeds the box length, $1 / m_{\pi}>L$, and the observables strongly depend on the topological sector. Our motivation to study this unphysical situation is that it allows for an evaluation of the Low Energy Constants (LEC) of the chiral Lagrangian with their values in infinite volume (unfortunately, quenching brings in logarithmic finite size effects [17]).

Random Matrix Theory (RMT) conjectures the densities $\rho_{n}^{(v)}(z)$ of the lowest Dirac eigenvalues $\lambda$ in the $\varepsilon$-regime [18], where $z:=\lambda \Sigma V, n=1,2, \ldots$ numerates the lowest non-zero eigenvalues and $v$ is the fermion index, which is identified with the topological charge [ฤ]. These conjectures hold to a good precision for the lowest $n$ and $|v|$, if $L$ exceeds a lower limit (a little more than $1 \mathrm{fm}$ ) [19]. Then the fit determines the scalar condensate $\Sigma$. Fig. 目 (left) shows our results for $\left\langle z_{1}\right\rangle$ with $D_{\mathrm{ov}-\mathrm{W}}$ and $D_{\mathrm{ov}-\mathrm{HF}}$, in $V=(1.48 \mathrm{fm})^{3} \times 2.96 \mathrm{fm}$. The RMT conjectures work best in the sectors $|v|=0,1,2$, and they provide precise values for $\Sigma$; for $D_{\mathrm{ov}-\mathrm{HF}}$ we obtain $\Sigma=(\mathbf{2 6 8}(\mathbf{2}) \mathbf{M e V})^{\mathbf{3}}$.
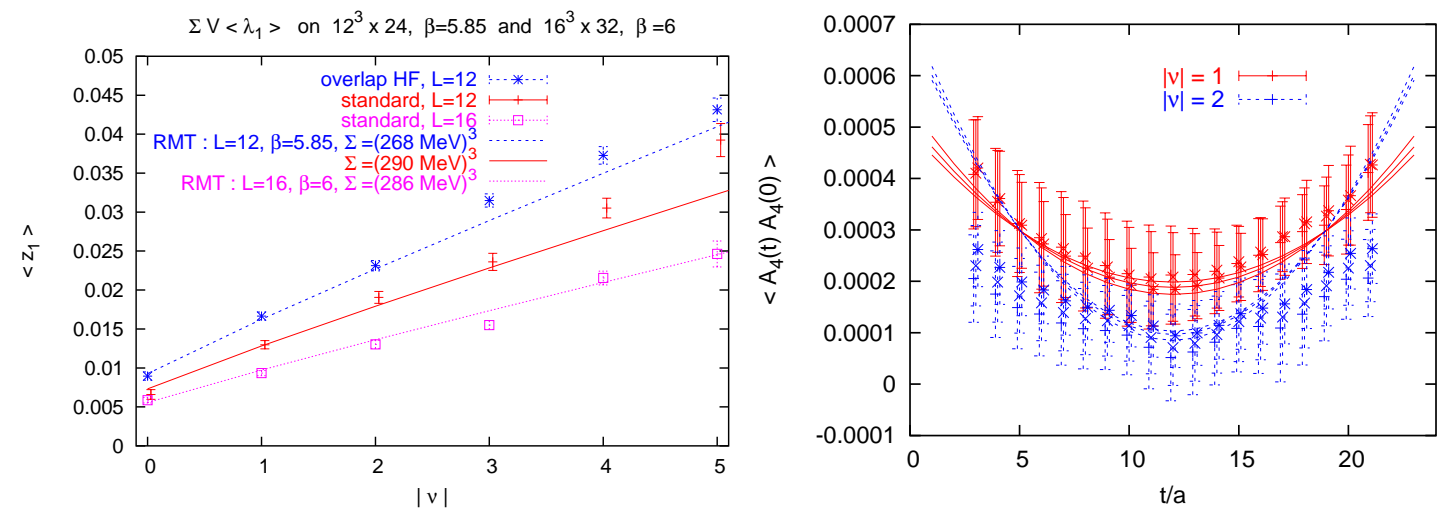

Figure 5: On the left: the lowest Dirac eigenvalue (re-scaled with $\Sigma V$ ) compared the the RMT predictions at the optimal value of $\Sigma$. On the right: preliminary results for the axial correlator at $a m_{q}=0.001,0.003$ and 0.005 in the sectors $|v|=1$ and 2, and fits to the formulae of quenched $\chi \mathrm{PT}$.

In quenched $\chi \mathrm{PT}$, the axial vector correlator depends in leading order only on the LEC $\Sigma$ and $F_{\pi}$ [20]. The prediction for $\left\langle A_{4}(t) A_{4}(0)\right\rangle$ (with $A_{\mu}(t):=\sum_{\vec{x}} \bar{\psi}(\vec{x}, t) \gamma_{5} \gamma_{\mu} \psi(\vec{x}, t)$ ) is a parabola with a minimum at $t=T / 2$, where $F_{\pi}^{2} / T$ enters as an additive constant. In a previous study we observed that $L$ should again be above $1 \mathrm{fm}$, and that the history in the sector $v=0$ may be plagued by 
spikes [21]; "Low Mode Averaging" was then invented as a remedy [22]. In the non-trivial sectors $\Sigma$ can hardly be determined, but $F_{\pi}$ can be evaluated. In Fig. 5 (right) we show preliminary results from three $m_{q}$ values in the $\varepsilon$-regime, in the sector $|v|=1$ and 2, with 10 propagators in each case. Inserting the RMT result for $\Sigma$, a global fit yields bare $F_{\pi}^{(0)}=(96 \pm 10) \mathrm{MeV}$, which is renormalised with $Z_{A}=1.17$ to $\mathbf{F}_{\pi}=(\mathbf{1 0 5} \pm \mathbf{1 3}) \mathbf{M e V}$, in agreement with other quenched results [22, 26, 12].
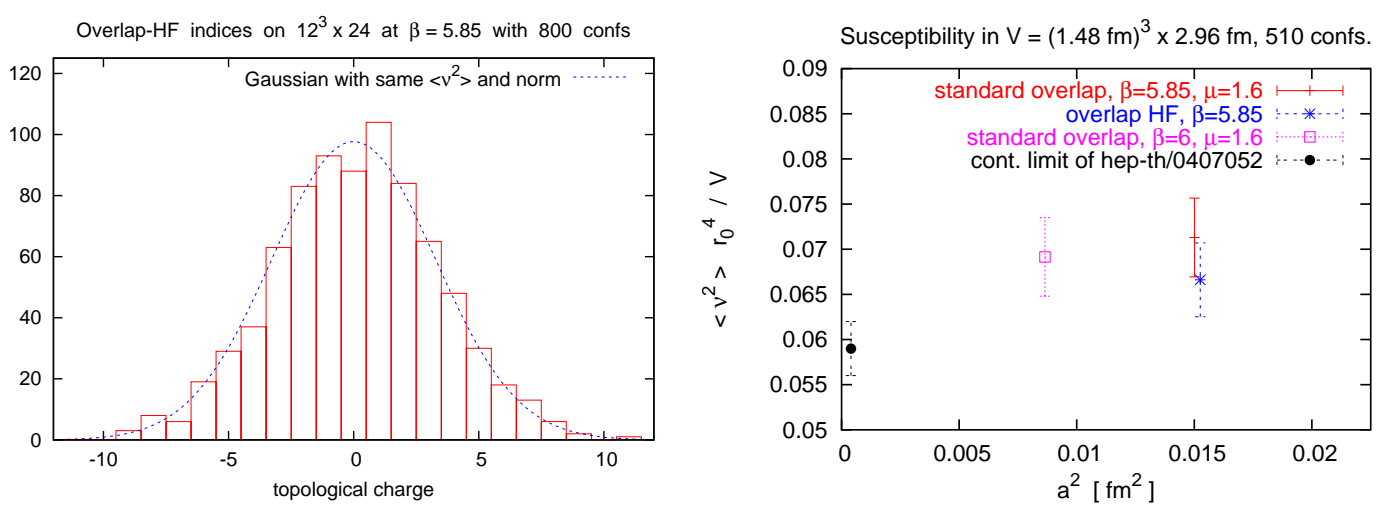

Figure 6: Histogram of overlap HF indices, and results for the topological susceptibility.

For 800 overlap HF indices we obtained the histogram in Fig. 6(left). The double peak reminds us of the question if parity could be broken [23], but the current statistics is of course not conclusive for this point. Fig. 6 (right) shows our results for the topological susceptibility, compared to the

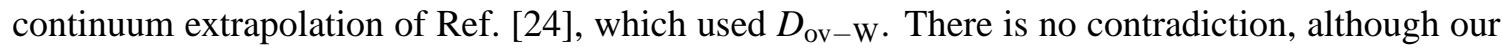
susceptibilities are somewhat larger. The $D_{\mathrm{ov}-\mathrm{HF}}$ result is closer to the value of Ref. [24] (for exactly the same configurations at $\beta=5.85$, with $\left.\left\langle\left|v_{\mathrm{ov}-\mathrm{W}}-v_{\mathrm{ov}-\mathrm{HF}}\right|\right\rangle \approx 0.8\right)[25]$.

\section{Conclusions}

The overlap HF operator provides better locality, and therefore chiral fermion on coarser lattices than the standard overlap operator. In the $p$-regime we gave results for $m_{\pi}, m_{\rho}$ and $F_{\pi}$. Compared to the standard overlap fermion, $m_{\mathrm{PCAC}}$ is closer to the bare quark mass $m_{q}$, hence $Z_{A}$ is much closer to 1 . In the $\varepsilon$-regime the confrontation with RMT yields a precise value for $\Sigma$, and from the axial correlation we extracted a preliminary result for $F_{\pi}$, which agrees (within the errors) with the chiral extrapolation of the direct measurement in the $p$-regime. In the $\varepsilon$-regime one may consider as an alternative solely the 0 -mode contributions to the mesonic correlators [26, 8, 25]. Finally we add that a topology conserving gauge action could be helpful in that regime [27].

We are indebted to M. Papinutto and C. Urbach for valuable numerical tools. We also thank them and B. Alles, L. Del Debbio, S. Dürr, H. Fukaya, K.-I. Nagai, K. Ogawa, L. Scorzato, A. Shindler, H. Stüben and $U$. Wenger for useful communications. This work was supported by the Deutsche Forschungsgemeinschaft through SFB/TR9-03. The computations were performed on the IBM p690 clusters of the HLRN "Norddeutscher Verbund für Hoch- und Höchstleistungsrechnen” (HLRN) and at NIC, Forschungszentrum Jülich.

\section{References}

[1] W. Bietenholz and U.-J. Wiese, Phys. Lett. B 378 (1996) 222 [hep-1at/9503022]; Nucl. Phys. B 464 (1996) 319 [hep-lat/9510026]. 
[2] W. Bietenholz, R. Brower, S. Chandrasekharan and U.-J. Wiese, Nucl. Phys. B (Proc. Suppl.) 53 (1997) 921 [hep-lat/9608068]. W. Bietenholz and U.-J. Wiese, Phys. Lett. B 426 (1998) 114 [hep-lat/9801022].

[3] H. Neuberger, Phys. Lett. B 417 (1998) 141 [hep-lat/9707022].

[4] P. Hasenfratz, V. Laliena and F. Niedermayer, Phys. Lett. B 427 (1998) 125 [hep-1at/9801021]. P. Hasenfratz, Nucl. Phys. B 525 (1998) 401 [hep-lat/ 9802007 ]. M. Lüscher, Phys. Lett. B428 (1998) 342 [hep-lat/9802011].

[5] D.H. Adams, Annals Phys. 296 (2002) 131 [hep-lat/9812003].

[6] D.H. Adams and W. Bietenholz, Eur. Phys. J. C 34 (2004) 245 [hep-lat / 0307022 ].

[7] W. Bietenholz, Eur. Phys. J. C 6 (1999) 537 [hep-lat/9803023]; hep-lat/ 0007017 ; Nucl. Phys. B 644 (2002) 223 [hep-lat/ 0204016 ]. W. Bietenholz and I. Hip, Nucl. Phys. B 570 (2000) 423 [hep-lat/9902019].

[8] S. Shcheredin, Ph.D. thesis, Berlin (2004) [hep-lat/0502001], and these Proc.

[9] P. Hernández, K. Jansen and M. Lüscher, Nucl. Phys. B 552 (1999) 363 [hep-lat/9808010].

[10] P. Hasenfratz et al., Nucl. Phys. B 643 (2002) 280 [hep-lat/ 0205010 ].

[11] $\chi$ LF Collaboration, JHEP 0412 (2004) 044 [hep-lat / 0411001 ].

[12] H. Fukaya, S. Hashimoto and K. Ogawa, hep-lat/0504018. K. Ogawa and S. Hashimoto, hep-lat/0505017.

[13] F. Berruto et al., Nucl. Phys. B (Proc. Suppl.) 129\&130 (2004) 471 [hep-lat/ 0310006 ].

[14] S. Dürr, C. Hoelbling and U. Wenger, hep-lat/0506027.

[15] G. Colangelo and S. Dürr, Eur. Phys. J. C 33 (2004) 543 [hep-lat/0311023].

[16] J. Gasser and H. Leutwyler, Phys. Lett. B 188 (1987) 477.

[17] P.H. Damgaard, Nucl. Phys. B 608 (2001) 162 [hep-lat/ 0105010 ].

[18] P.H. Damgaard and S.M. Nishigaki, Phys. Rev. D 63 (2001) 045012 [hep-th/ 0006111 ].

[19] W. Bietenholz, K. Jansen and S. Shcheredin, JHEP 07 (2003) 033 [hep-lat/0306022]. L. Giusti, M. Lüscher, P. Weisz and H. Wittig, JHEP 11 (2003) 023 [hep-lat/ 0309189 ]. QCDSF-UKQCD Collaboration, Nucl. Phys. B (Proc. Suppl.) 129\&130 (2004) 456 [hep-lat/ 0310028 ].

[20] P.H. Damgaard, P. Hernández, K. Jansen, M. Laine and L. Lellouch, Nucl. Phys. B 656 (2003) 226 [hep-lat/0211020].

[21] W. Bietenholz, T. Chiarappa, K. Jansen, K.-I. Nagai and S. Shcheredin, JHEP 0402 (2004) 023 [hep-lat/0311012].

[22] L. Giusti et al., JHEP 0404 (2004) 013 [hep-lat / 0402002 ]. P. Hernández et al., these Proc.

[23] V. Azcoiti and A. Galante, Phys. Rev. Lett. 83 (1999) 1518 [hep-th/9901068]. B. Alles, M. D'Elia and A. Di Giacomo, Phys. Rev. D 71 (2005) 034503 [hep-lat/ 0411035$].$

[24] L. Del Debbio, L. Giusti and C. Pica, Phys. Rev. Lett. 94 (2005) 032003 [hep-th/ 0407052 ].

[25] W. Bietenholz and S. Shcheredin, hep-lat/0502010.

[26] L. Giusti et al., JHEP 0401 (2004) 003 [hep-lat/0312012]. S. Necco et al., these Proc.

[27] H. Fukaya and T. Onogi, Phys. Rev. D 68 (2003) 074503 [hep-lat/ 030500 4]. S. Shcheredin et al., Nucl. Phys. B (Proc. Suppl.) 140 (2005) 779 [hep-lat/ 0409073 ]. W. Bietenholz et al., hep-lat/ 0412017 . H. Matsufuru et al., these Proc. K.-I. Nagai et al., these Proc. 